\title{
Sudden Unexpected Death by Myocardial Infarction Simulating Drowning: A Case Report
}

\author{
Enow Orock GE ${ }^{1 *}$, Njouendou $\mathrm{JA}^{1}$ and Obenson $\mathrm{K}^{2}$ \\ ${ }^{1}$ Department of Pathology, Faculty of Health Sciences, University of Buea, Cameroon \\ 2 Department of Laboratory Medicine, Saint John Regional Hospital, Saint John, New Brunswick, Canada
}

*Corresponding author: Enow Orock GE, Department of Pathology, Faculty of Health Sciences, University of Buea, Cameroon, Tel: 237-67771 6045; E-mail: enowrock24@yahoo.com

Received date: November 13, 2018; Accepted date: November 20, 2018; Published date: November 27, 2018

Copyright: (c) 2018 Enow Orock GE, et al. This is an open-access article distributed under the terms of the Creative Commons Attribution License, which permits unrestricted use, distribution, and reproduction in any medium, provided the original author and source are credited.

\begin{abstract}
We report the case of a middle-aged male working on board a ship. His body was found floating in the river, near shoreline, by security officials. A postmortem on the decomposed body revealed a recent myocardial infarction. Comorbid conditions included coronary atheromatous disease and long term tobacco smoking. Not all bodies found dead in water are likely to have drowned. This case demonstrates the importance of a thorough forensic investigation, particularly in histological terms, in subjects found dead in water, in order to ascertain the real cause of death, which may not be always assigned to asphyxia by drowning.
\end{abstract}

Keywords: Death; Drowning; Autopsy; Myocardial Infarction

\section{Introduction}

Determining the cause of death in bodies found in water is challenging [1]. Only after a thorough death investigation and complete autopsy the pathologist certify a death as being from asphyxia due to drowning, since it is a diagnosis of exclusion. However in spite of the proven utility of the autopsy to medical and judicial procedures, the procedure is infrequently requested even when indicated [2]. Autopsy rates continue to fall all over the world. The situation is worse in developing countries $[2,3]$ where cultural beliefs and practices, lack of qualified personnel and poverty limit its application [3].

For several years, the Cameroon Society of Pathology and Cancerology (previously the Cameroon Society of Pathology) has pushed unsuccessfully for legislation to regulate the practice of autopsy in Cameroon. At a rate of 1 autopsy per 100 deaths in 2005 and 4-5/100 in 2018; autopsy rate in Cameroon is still very low [2] though it is similar to rates reported in Nigeria [4]. It is important that all stake holders-medical, legislative, judicial, traditional and administrative are sensitized to the importance of the autopsy in certifying the exact cause of death in questionable circumstances [4].

Bodies found dead in water are not routinely autopsied in Cameroon. This case was referred for autopsy for various peculiar reasons. Firstly, the deceased was an expatriate and diplomatic pressure was mounted on the government for a full death investigation. Secondly, this death occurred in a conflict zone where separatist fighting the government forces sometimes target civilians, most especially expatriates, for kidnap and/or assassination. The impression of this death by the general public, security services and home country of deceased was overwhelmingly in favour of a violent death, likely a homicide. Were it not for an autopsy, this case should have passed for drowning or any other homicidal cause. In fact it should have passed for anything but a natural death.
Our main vision is for a legislation that obliges all deaths in suspicious circumstances in or out of hospital to undergo autopsy in our community. This explains why we report this spectacular case of sudden unexpected death by myocardial infarction simulating drowning.

\section{Case Report}

Decedent was a middle-aged male expatriate who appeared to be the stated age of 55 years and employed as a ship engineer. He was last known to be on duty at sea 3 days previously when he went missing. A search team recovered his fully clothed "drowned" body by the river bank downstream. A coroner's inquest into the death was instituted and the body was sent to the department of pathology at the Buea Regional Hospital for autopsy. The body was recovered in an area of active conflict which posed additional diplomatic and socio-political challenges.

He was on unspecified medication for heart disease and was a 'heavy' smoker of long duration.

External examination showed the body of a middle-aged male in advanced decomposition.

There were no obvious signs of physical trauma or catastrophic hemorrhage. Internal examination was significant for bilateral lung collapse with patent and dry airways. No fluid exuded from the lungs even on digital pressure (Figure 1). The heart looked normal and weighed about $400 \mathrm{~g}$ with the left ventricular wall measuring $2.0 \mathrm{~cm}$ in thickness. The anterior descending left coronary artery showed a thrombus on an atheromatous plaque at about $1.8 \mathrm{~cm}$ distance from the arterial origin causing approximately $70 \%$ occlusion of the artery.

Dissection of the heart revealed a massive sub endocardial infarct involving the left ventricle to the cardiac apex (Figure 2). Paraffinembedded heart samples were selected from the left ventricular wall and samples were sectioned at $5 \mu \mathrm{m}$ and stained with Hematoxylin and Eosin (H\&E) then examined under the light microscope. Microscopic examination showed inter fiber infiltration by neutrophils and nuclear/ 
cytoplasmic degenerative changes consistent with a recent infarction. No previous infarcts were seen. A diagnosis of an acute massive fatal Myocardial Infarction (AMI) was made due to a coronary thrombus in a background of ischemic cardiac disease and long term tobacco use. The rest of the examination was unremarkable. Due to logistical reasons, toxicological analyses were not performed. The manner of death was deemed natural.

\section{Discussion}

The majority of bodies found in water are typically determined to have died from asphyxia due to drowning. Many have associated ischemic cardiovascular disease, either as a contributing factor, or the underlying causative factor [5]. Some of them are due to homicidal circumstances while others are natural. In 1999 Modell et al. deemed it appropriate to apply to the latter the notion of "drowning without aspiration" [5]. In this case, further investigations provided evidence that underlying natural disease may have contributed to and/or caused death in water.

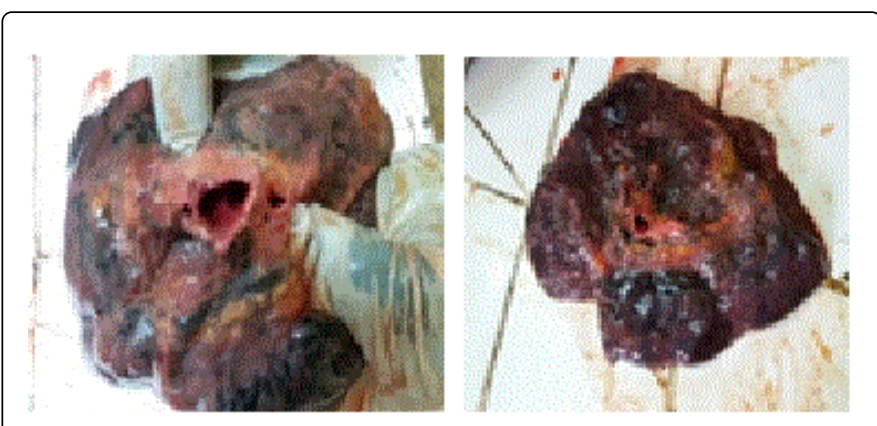

Figure 1: Severely anthracotic lung showing dry and clear airway even on digital pressure to the lungs.

Sudden/unexpected death may occur in apparently healthy individuals, even those without any known disease. The majority of these deaths are related to cardiovascular disease which commonly occur in elderly males [6] like in our case, though there was no confirmed history of heart disease. Moreover this case occurred in a very precarious socio political context. The area where this occurred was in active conflict at the time. Apart from the medico-legal challenges, this case posed obvious diplomatic and socio-political challenges.

Even in advanced state of decomposition it is sometimes possible to find macroscopic evidence of cause of death $[7,8]$. Travoula in a report [9] found 29/187 immersed cases died from non-drowning causes and 23 of these died from cardiac causes. Our main finding in this case was in the lungs and the heart. The predisposing risk was an atheromatous plaque in the wall of the left coronary artery causing about $70 \%$ occlusion. This is consistent with reports that have shown the left coronary to be more susceptible to stenotic artherosclerotic lesions $[10,11]$. Occlusive artherosclerotic plaques in the coronary arteries are often found within the first $2 \mathrm{~cm}$ of the artery [9]. In our case the plaque was found at $1.8 \mathrm{~cm}$ from the arterial origin, the heart weighed about $400 \mathrm{~g}$ and the left ventricle measured $2.0 \mathrm{~cm}$ in thickness. Kortelainen [12] reports that myocardial infarction is reported to be the immediate cause of death in $14.4 \%$ of men and associated with increased heart size in the male sex. . In our case there was no increase in the size of the heart. Macroscopically a fresh recent left ventricular sub endocardial infarct spanning the entire left ventricular cardiac muscle thickness to the sub epicardium was found.

In one case of a deep sea diver, death was attributed to arrhythmia due to breath- holding [1].

Comorbidities in cardiac deaths include age, body size and cardiac parameters including cardiomyopathies, coronary artery disease, an acute myocardial infarction or coronary heart disease without infarction. Other confounding/contributory factors include diabetes, alcohol consumption and smoking [12]. Luminal narrowing of the coronary artery from arterosclerosis, atheroma, with or without a thrombus is a contributory factor to most myocardial deaths $[10,11,13]$. We found a coronary luminal narrowing of $70 \%$ in our victim.

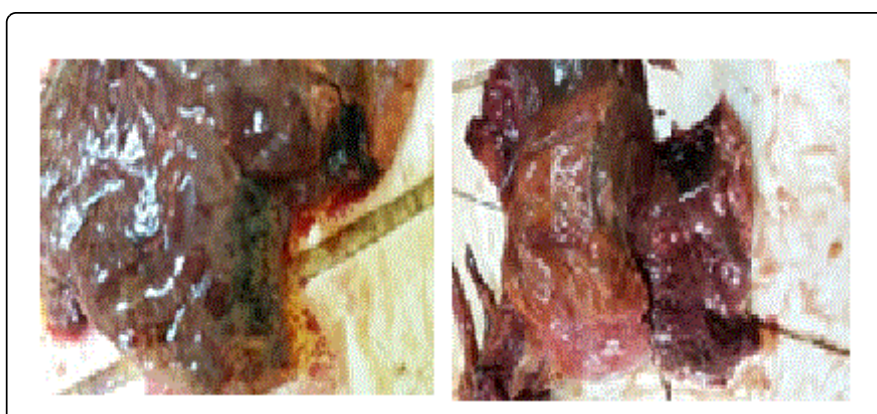

Figure 2: Transverse sections through left ventricular wall to cardiac apex showing recent infarction

Dissection of the lungs revealed a clean and dry airway with no exudation of fluid on digital pressure to the lungs (Figure 1). Drowning victims inhale water which inundates and fills the lung airways. The lungs are distended and the airway is filled with frothy fluid which exudes on pressure. Sometimes aquatic flora like algae can be found in lung fluids. These later which may serve as forensic evidence assist in identifying the water where drowning occurred. None of these was found in our case. In an unpublished case about a decade ago of suspected homicide in a body found dead in water in this community, the author was part of a forensic team that found algae and other water flora in the lungs of the victim to conclude this as a case of drowning. Microscopic examination showed inter fiber infiltration by neutrophils and nuclear/cytoplasmic degenerative changes consistent with a recent infarction as reported in literature (Figure 3) [14].

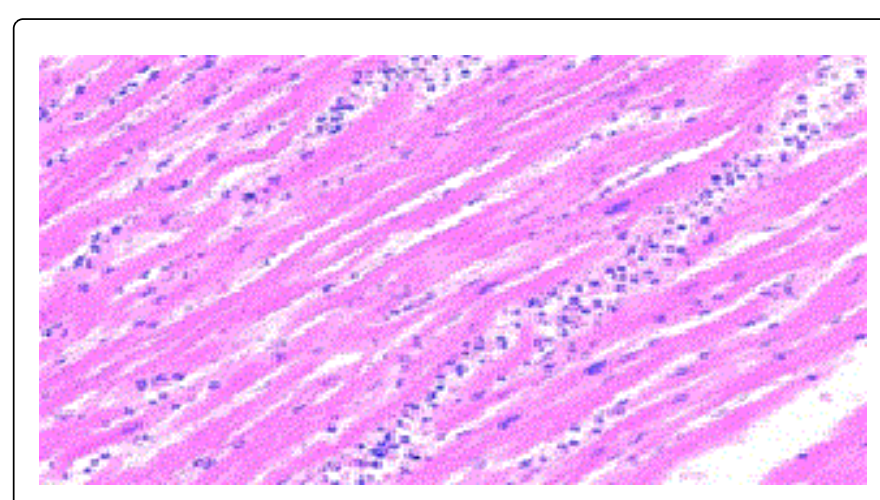

Figure 3: Histology showing acute myocardial infarction 
Citation: Enow Orock GE, Njouendou JA, Obenson K (2018) Sudden Unexpected Death by Myocardial Infarction Simulating Drowning: A Case Report. J Forensic Med 3: 126. doi:10.4172/2472-1026.1000126

Page 3 of 3

\section{Conclusion}

An autopsy is vital in the determination of the actual cause of any death. This procedure is even more indicated in suspicious and/or unnatural death circumstance. It is hoped that this report will contribute to lay emphasis and hasten elaboration of legislation regulating the practice of autopsy in our community given its importance in medical jurisprudence and forensic science.

\section{References}

1. Elvira VS, Cristina M, Luigi C, Stefania Z, Giulio C (2016) Sudden death in water: Diagnostic challenges. Case Report. Egypt J Forensic Sci 6: 22-25.

2. Enow OGE, Assob NCJ, Pisoh CT, Masumbe PS, Sando Z, et al. (2009) Contribution of autopsy to medical practice in Cameroon: A ten year review. Clin Mother Child Health 6: 1091-1094.

3. Obenson K, Enow OG (2017) An overview of the challenges facing death investigation systems in certain resource-limited countries. J Forensic Leg Med 50: 58-62.

4. Ekpo MD, Abudu EK, Umanah IN, Onwuezobe IA (2011) An audit of medical autopsy: Experience at the University of Uyo Teaching Hospital (UUTH), Nigeria. Indian J Med Sci 6: 502-509.

5. Schwameis M, Schober A, Schörgenhofer C, Sperr WR, Schöchl H, et al. (2015) Asphyxia by drowning induces massive bleeding due to hyperfibrinolytic disseminated intravascular coagulation. Crit Care Med 43: 2394-2402.

6. Prateek R, Nagesh KR, Mukta RP (2006) Sudden Cardiac death due to Myocardial Infarction in a young Female- A Case report. J Indian Acad Forensic Med 28: 140-141.

7. (2011) Decomposing body found at McKoy Lane. Jamaica Observer.

8. Ambade VN, Keoliya AN, Deokar RB, Dixit PG (2011) Decomposed bodies-still an unrewarding autopsy? J Forensic Leg Med 18: 101-106.

9. Tavroula S, Apadodima AP, Mmanouil E, Akelliadis S, Anagiotis P, et al. (2007) Cardiovascular disease and drowning: Autopsy and laboratory findings. Hellenic J Cardiol 48: 198-205.

10. Xianchi L, Min L, Rongrong S, Zeng Y, Shuang C, et al. (2016) Atherosclerotic coronary artery disease: The accuracy of measures to diagnose preclinical atherosclerosis. Exp Ther Med 12: 2899-2902.

11. Simon A, Chironi G, Levenson J (2007) Comparative performance of subclinical atherosclerosis tests in predicting coronary heart disease in asymptomatic individuals. Eur Heart J 28: 2967-2971.

12. Kortelainen ML, Särkioja T (2002) Myocardial infarction and coronary pathology in severely obese people examined at autopsy. Int J Obes 26: 73-79.

13. Otsuka F, Yasuda S, Noguchi T, Ishibashi-Ueda H (2016) Pathology of coronary atherosclerosis and thrombosis. Cardiovasc Diagn Ther 6: 396-408.

14. White HD, Chew DP (2008) Acute myocardial infarction. Lancet 372: 570-584. 